\title{
Ulam type stability problems for alternative homomorphisms
}

\author{
Sin-Ei Takahasi ${ }^{* *}$, Makoto Tsukada ${ }^{1}$, Takeshi Miura ${ }^{2}$, Hiroyuki Takagi ${ }^{3}$ and Kotaro Tanahashi ${ }^{4}$
}

"Correspondence:

sin_ei1@yahoo.co.jp

'Department of Information

Sciences, Toho University,

Funabashi, 274-8501, Japan

Full list of author information is

available at the end of the article

\begin{abstract}
We introduce an alternative homomorphism with respect to binary operations and investigate the Ulam type stability problem for such a mapping. The obtained results apply to Ulam type stability problems for several important functional equations.
\end{abstract}

MSC: Primary 39B82; secondary 47H10

Keywords: Ulam type stability; homomorphism; binary operation; fixed point theorem

\section{Introduction}

In 1940, SM Ulam proposed the following stability problem: Given an approximately additive mapping, can one find the strictly additive mapping near it? A year later, DH Hyers gave an affirmative answer to this problem for additive mappings between Banach spaces. Subsequently many mathematicians came to deal with this problem $(c f .[1-5])$.

We introduce an alternative homomorphism from a set $X$ with two binary operations o and $*$ to another set $E$ with two binary operations $\diamond$ and $\star$ defined by

$$
f(x \circ y) \star f(x * y)=f(x) \diamond f(y) \quad(\forall x, y \in X),
$$

and we investigate the Ulam type stability problem for such a mapping when $E$ is a complete metric space. In particular, if $s \star t=s$ for all $s, t \in E$, then our results imply the stability results obtained in [6]. Also the method used in the paper have already applied for some other equations (cf. [7-15]).

\section{One consequence of Banach's fixed point theorem}

A fixed point theorem has played an important role in the stability problem (cf. [16]). The authors used an easy consequence of Banach's fixed point theorem in [6]. It will serve again in this paper. Here we review it.

Let $X$ be a set and $(E, d)$ a complete metric space. Fix two mappings $f: X \rightarrow E$ and $\varphi: X \rightarrow \mathbb{R}^{+}$, where $\mathbb{R}^{+}$denotes the set of all nonnegative real numbers. Denote by $\Delta_{f, \varphi}$ the set of all mappings $u: X \rightarrow E$ such that there exists a finite constant $K_{u}$ satisfying

$$
d(u(x), f(x)) \leq K_{u} \varphi(x) \quad(\forall x \in X) .
$$

O2014 Takahasi et al.; licensee Springer. This is an Open Access article distributed under the terms of the Creative Commons Attribution License (http://creativecommons.org/licenses/by/2.0), which permits unrestricted use, distribution, and reproduction in any medium, provided the original work is properly cited. 
For any $u, v \in \Delta_{f, \varphi}$, we define

$$
\rho_{f, \varphi}(u, v)=\inf \{K \geq 0: d(u(x), v(x)) \leq K \varphi(x)(\forall x \in X)\} .
$$

Then $\left(\Delta_{f, \varphi}, \rho_{f, \varphi}\right)$ is a complete metric space which contains $f$.

Now, fix three mappings $\sigma: X \rightarrow X, \tau: E \rightarrow E$ and $\varepsilon: X \times X \rightarrow \mathbb{R}^{+}$. For any mapping $u: X \rightarrow E$, we define the mapping $T_{\sigma, \tau} u: X \rightarrow E$ by

$$
\left(T_{\sigma, \tau} u\right)(x)=\tau(u(\sigma x)) \quad(x \in X)
$$

Also, we consider three quantities:

$$
\begin{aligned}
& \alpha_{\sigma, \varepsilon}=\inf \{K \geq 0: \varepsilon(\sigma x, \sigma y) \leq K \varepsilon(x, y)(x, y \in X)\}, \\
& \beta_{\sigma, \varphi}=\inf \{K \geq 0: \varphi(\sigma x) \leq K \varphi(x)(x \in X)\}, \\
& \gamma_{\tau}=\inf \{K \geq 0: d(\tau s, \tau t) \leq K d(s, t)(s, t \in E)\} .
\end{aligned}
$$

If $\alpha_{\sigma, \varepsilon}<\infty, \beta_{\sigma, \varphi}<\infty$ and $\gamma_{\tau}<\infty$, then we have

$$
\begin{aligned}
& \varepsilon(\sigma x, \sigma y) \leq \alpha_{\sigma, \varepsilon} \varepsilon(x, y) \quad(\forall x, y \in X), \\
& \varphi(\sigma x) \leq \beta_{\sigma, \varphi} \varphi(x) \quad(\forall x \in X), \\
& d(\tau s, \tau t) \leq \gamma_{\tau} d(s, t) \quad(\forall s, t \in E),
\end{aligned}
$$

respectively. We will use these inequalities throughout this paper.

We now state our fixed point theorem.

Lemma A ([6, Proposition 2.1]) Let $X$ be a set and $(E, d)$ a complete metric space. Suppose that four mappings $f: X \rightarrow E, \varphi: X \rightarrow \mathbb{R}^{+}, \sigma: X \rightarrow X$ and $\tau: E \rightarrow E$ satisfy

$$
T_{\sigma, \tau} f \in \Delta_{f, \varphi}, \quad \beta_{\sigma, \varphi}<\infty, \quad \gamma_{\tau}<\infty \quad \text { and } \quad \beta_{\sigma, \varphi} \gamma_{\tau}<1
$$

Then $T_{\sigma, \tau}\left(\Delta_{f, \varphi}\right) \subseteq \Delta_{f, \varphi}$ and $T_{\sigma, \tau}$ has a unique fixed point $f_{\infty}$ in $\Delta_{f, \varphi}$. Moreover,

$$
\lim _{n \rightarrow \infty} d\left(\left(T_{\sigma, \tau}^{n} f\right)(x), f_{\infty}(x)\right)=0 \quad \text { and } \quad d\left(f(x), f_{\infty}(x)\right) \leq \frac{\rho_{f, \varphi}\left(T_{\sigma, \tau} f, f\right)}{1-\beta_{\sigma, \varphi} \gamma_{\tau}} \varphi(x)
$$

for all $x \in X$.

\section{A stability of alternative homomorphisms}

Let $(X, \circ, *)$ be a set $X$ with two binary operations $\circ$ and $*$. Let $(E, d, \diamond, \star)$ be a complete metric space $(E, d)$ with two binary operations $\diamond$ and $\star$. Given $f: X \rightarrow E$, we consider the following commutative diagram:

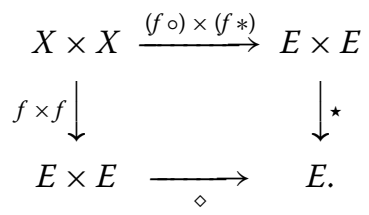


This means that

$$
f(x \circ y) \star f(x * y)=f(x) \diamond f(y) \quad(\forall x, y \in X)
$$

In particular, if $s \star t=s$ for all $s, t \in E$, then (1) and (2) become

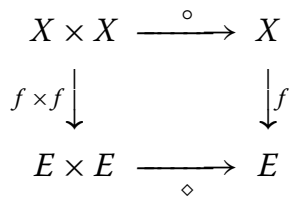

and

$$
f(x \circ y)=f(x) \diamond f(y) \quad(\forall x, y \in X)
$$

In other words, $f$ is a homomorphism from $X$ to $E$. Thus, if a mapping $f: X \rightarrow E$ satisfies (2), then we say that $f$ is an alternative homomorphism.

In this section, we establish two general settings, on which we can give an affirmative answer to the Ulam type stability problem for the commutative diagram (1). These settings have a property such as duality, that is, each of them works as a complement of the other.

Let us describe the first setting. For $\varepsilon: X \times X \rightarrow \mathbb{R}^{+}$and $\delta: X \rightarrow \mathbb{R}^{+}$, we consider the following three conditions:

(i) The square operator $x \mapsto x \circ x$ is an automorphism of $X$ with respect to $\circ$ and $*$. We denote by $\sigma$ the inverse mapping of this automorphism.

(ii) The binary operations $\diamond$ and $\star$ on $E$ are continuous. The square operator $\tau: s \mapsto s \diamond s$ is an endomorphism of $E$ with respect to $\diamond$ and $\star$.

(iii) $\alpha \equiv \alpha_{\sigma, \varepsilon}<\infty, \beta \equiv \beta_{\sigma, \delta}<\infty, \gamma \equiv \gamma_{\tau}<\infty$ and $\gamma \max \{\alpha, \beta\}<1$.

Under the above conditions, we show the Ulam type stability for the commutative diagram (1), as follows.

Theorem 1 Let $(X, \circ, *)$ and $(E, d, \diamond, \star)$ be as above. Suppose that four mappings $\sigma: X \rightarrow X$, $\tau: E \rightarrow E, \varepsilon: X \times X \rightarrow \mathbb{R}^{+}$and $\delta: X \rightarrow \mathbb{R}^{+}$satisfy (i), (ii), and (iii). If a mapping $f: X \rightarrow E$ satisfies

$$
\begin{aligned}
& d(f(x \circ y) \star f(x * y), f(x) \diamond f(y)) \leq \varepsilon(x, y) \quad(\forall x, y \in X), \\
& d(f(x) \star f(\sigma x * \sigma x), f(x)) \leq \delta(x) \quad(\forall x \in X),
\end{aligned}
$$

then there exists a mapping $f_{\infty}: X \rightarrow$ E such that

$$
\begin{aligned}
& f_{\infty}(x \circ y) \star f_{\infty}(x * y)=f_{\infty}(x) \diamond f_{\infty}(y) \quad(\forall x, y \in X), \\
& f_{\infty}(x) \star f_{\infty}(\sigma x * \sigma x)=f_{\infty}(x) \quad(\forall x \in X), \\
& d\left(f(x), f_{\infty}(x)\right) \leq \frac{\alpha \varepsilon(x, x)+\delta(x)}{1-\gamma \max \{\alpha, \beta\}} \quad(\forall x \in X) .
\end{aligned}
$$

Moreover, if a mapping $g: X \rightarrow E$ satisfies (5), (6), and

$$
\exists K_{g} \geq 0: d(f(x), g(x)) \leq K_{g}\{\alpha \varepsilon(x, x)+\delta(x)\} \quad(\forall x \in X),
$$

then $g=f_{\infty}$. 
Proof For simplicity, we write $T=T_{\sigma, \tau}$. We note that $\alpha, \beta$, and $\gamma$ are finite by (iii). Suppose that $f: X \rightarrow E$ satisfies (3) and (4). Put $\varphi(x)=\alpha \varepsilon(x, x)+\delta(x)$ for all $x \in X$. To apply Lemma A to $f$ and $\varphi$, we first observe that $T f \in \Delta_{f, \varphi}$. Fix $x \in X$. Replacing $x$ and $y$ in (3) by $\sigma x$, we get

$$
d(f(\sigma x \circ \sigma x) \star f(\sigma x * \sigma x), f(\sigma x) \diamond f(\sigma x)) \leq \varepsilon(\sigma x, \sigma x)
$$

Since

$$
\begin{aligned}
& \sigma x \circ \sigma x=\sigma^{-1}(\sigma x)=x, \\
& f(\sigma x) \diamond f(\sigma x)=\tau(f(\sigma x))=(T f)(x),
\end{aligned}
$$

and

$$
\varepsilon(\sigma x, \sigma x) \leq \alpha \varepsilon(x, x)
$$

it follows that

$$
d(f(x) \star f(\sigma x * \sigma x),(T f)(x)) \leq \alpha \varepsilon(x, x) .
$$

Using this and (4), we have

$$
\begin{aligned}
d((T f)(x), f(x)) & \leq d((T f)(x), f(x) \star f(\sigma x * \sigma x))+d(f(x) \star f(\sigma x * \sigma x), f(x)) \\
& \leq \alpha \varepsilon(x, x)+\delta(x) \\
& =\varphi(x) .
\end{aligned}
$$

Hence $T f \in \Delta_{f, \varphi}$ and $\rho_{f, \varphi}(T f, f) \leq 1$.

We next estimate the quantity $\beta_{\sigma, \varphi}$. For $x \in X$, we have

$$
\begin{aligned}
\varphi(\sigma x) & =\alpha \varepsilon(\sigma x, \sigma x)+\delta(\sigma x) \\
& \leq \alpha^{2} \varepsilon(x, x)+\beta \delta(x) \\
& \leq \max \{\alpha, \beta\}(\alpha \varepsilon(x, x)+\delta(x)) \\
& =\max \{\alpha, \beta\} \varphi(x) .
\end{aligned}
$$

Hence $\beta_{\sigma, \varphi} \leq \max \{\alpha, \beta\}$ and $\beta_{\sigma, \varphi} \gamma_{\tau} \leq \gamma \max \{\alpha, \beta\}<1$ by (iii).

Thus we can apply Lemma A. As a consequence, $T$ has a unique fixed point $f_{\infty} \in \Delta_{f, \varphi}$. Moreover,

$$
\lim _{n \rightarrow \infty} d\left(\left(T^{n} f\right)(x), f_{\infty}(x)\right)=0
$$

and

$$
d\left(f(x), f_{\infty}(x)\right) \leq \frac{\rho_{f, \varphi}(T f, f)}{1-\beta_{\sigma, \varphi} \gamma_{\tau}} \varphi(x)
$$

for all $x \in X$. Since $\rho_{f, \varphi}(T f, f) \leq 1$ and $\beta_{\sigma, \varphi} \gamma_{\tau} \leq \gamma \max \{\alpha, \beta\}<1,(10)$ implies (7). 
Here we show (5). If $x, y \in X$ and $n \in \mathbb{N}$, then we have

$$
\begin{aligned}
& d\left(f_{\infty}(x \circ y) \star f_{\infty}(x * y), f_{\infty}(x) \diamond f_{\infty}(y)\right) \\
& \leq d\left(f_{\infty}(x \circ y) \star f_{\infty}(x * y),\left(T^{n} f\right)(x \circ y) \star\left(T^{n} f\right)(x * y)\right) \\
& \quad+d\left(\left(T^{n} f\right)(x \circ y) \star\left(T^{n} f\right)(x * y),\left(T^{n} f\right)(x) \diamond\left(T^{n} f\right)(y)\right) \\
& \quad+d\left(\left(T^{n} f\right)(x) \diamond\left(T^{n} f\right)(y), f_{\infty}(x) \diamond f_{\infty}(y)\right) .
\end{aligned}
$$

We will see that the right hand side of (11) tends to 0 as $n \rightarrow \infty$. The first and third terms on the right hand side tend to 0 as $n \rightarrow \infty$, because of (9) and the continuity of $\star$ and $\diamond$ in (ii). Moreover, the second term, say $A_{n}(x, y)$, is estimated as follows: By (i), (ii), and (3), we have

$$
\begin{aligned}
A_{n}(x, y) & =d\left(\tau^{n}\left(f\left(\sigma^{n}(x \circ y)\right)\right) \star \tau^{n}\left(f\left(\sigma^{n}(x * y)\right)\right), \tau^{n}\left(f\left(\sigma^{n} x\right)\right) \diamond \tau^{n}\left(f\left(\sigma^{n} y\right)\right)\right) \\
& =d\left(\tau^{n}\left(f\left(\sigma^{n} x \circ \sigma^{n} y\right)\right) \star \tau^{n}\left(f\left(\sigma^{n} x * \sigma^{n} y\right)\right), \tau^{n}\left(f\left(\sigma^{n} x\right) \diamond f\left(\sigma^{n} y\right)\right)\right) \\
& =d\left(\tau^{n}\left(f\left(\sigma^{n} x \circ \sigma^{n} y\right) \star f\left(\sigma^{n} x * \sigma^{n} y\right)\right), \tau^{n}\left(f\left(\sigma^{n} x\right) \diamond f\left(\sigma^{n} y\right)\right)\right) \\
& \leq \gamma^{n} d\left(f\left(\sigma^{n} x \circ \sigma^{n} y\right) \star f\left(\sigma^{n} x * \sigma^{n} y\right), f\left(\sigma^{n} x\right) \diamond f\left(\sigma^{n} y\right)\right) \\
& \leq \gamma^{n} \varepsilon\left(\sigma^{n} x, \sigma^{n} y\right) \\
& \leq \gamma^{n} \alpha^{n} \varepsilon(x, y),
\end{aligned}
$$

where $\tau^{n}$ and $\sigma^{n}$ denote the $n$-fold compositions of endomorphisms $\tau$ and $\sigma$, respectively. Since $\gamma \alpha<1$ by (iii), it follows that $A_{n}(x, y) \rightarrow 0$ as $n \rightarrow \infty$. Thus the right hand side of (11) tends to 0 , and we obtain (5).

Next, we show (6). For $x \in X$, we replace $x$ and $y$ in (5) by $\sigma x$ to get

$$
f_{\infty}(\sigma x \circ \sigma x) \star f_{\infty}(\sigma x * \sigma x)=f_{\infty}(\sigma x) \diamond f_{\infty}(\sigma x)
$$

Since $\sigma x \circ \sigma x=x$ and

$$
f_{\infty}(\sigma x) \diamond f_{\infty}(\sigma x)=\tau\left(f_{\infty}(\sigma x)\right)=\left(T f_{\infty}\right)(x)=f_{\infty}(x)
$$

we obtain (6).

Finally, we show the last statement. Since $g$ satisfies (5) and (6), we have

$$
\begin{aligned}
(T g)(x) & =\tau(g(\sigma x))=g(\sigma x) \diamond g(\sigma x) \\
& =g(\sigma x \circ \sigma x) \star g(\sigma x * \sigma x) \\
& =g(x) \star g(\sigma x * \sigma x) \\
& =g(x)
\end{aligned}
$$

for all $x \in X$. This says that $g$ is a fixed point of $T$. Also, by (8), we have $g \in \Delta_{f, \varphi}$. Thus the uniqueness of a fixed point of $T$ in $\Delta_{f, \varphi}$ implies that $g=f_{\infty}$. 
The next corollary is obtained in [6].

Corollary 1 ([6, Corollary 3.2]) Let $X$ be a set with a binary operation $\circ$ such that the square operation $x \mapsto x \circ x$ is an automorphism of $X$ with respect to $\circ$ and $E$ a complete metric space with a continuous binary operation $\diamond$ such that the square operation $\tau: s \mapsto$ $s \diamond s$ is an endomorphism of $E$ with respect to $\diamond$. Let $\varepsilon: X \times X \rightarrow \mathbb{R}^{+}$and suppose that $\alpha \equiv \alpha_{\sigma, \varepsilon}<\infty, \gamma \equiv \gamma_{\tau}<\infty$ and $\gamma \alpha<1$, where $\sigma$ denotes the inverse mapping of the square operation $x \mapsto x \circ x$. If a mapping $f: X \rightarrow E$ satisfies

$$
d(f(x \circ y), f(x) \diamond f(y)) \leq \varepsilon(x, y) \quad(\forall x, y \in X)
$$

then there exists a unique mapping $f_{\infty}: X \rightarrow E$ such that

$$
f_{\infty}(x \circ y)=f_{\infty}(x) \diamond f_{\infty}(y) \quad \text { and } \quad d\left(f(x), f_{\infty}(x)\right) \leq \frac{\alpha}{1-\alpha \gamma} \varepsilon(x, x)
$$

for all $x, y \in X$.

Proof Consider the case that $*=\circ$ and $s \star t=s$ for $s, t \in E$, in Theorem 1. In this case, $\tau$ is clearly an endomorphism of $E$ with respect to $\star$. Therefore the corollary follows immediately from Theorem 1 with $\delta=0$.

Now we turn to another setting. Let $(X, \circ, *)$ and $(E, d, \diamond, \star)$ be as in the first part of this section. For $\varepsilon: X \times X \rightarrow \mathbb{R}^{+}$and $\delta: X \rightarrow \mathbb{R}^{+}$, we consider the following three conditions:

(iv) The square operator $\tilde{\sigma}: x \mapsto x \circ x$ is an endomorphism of $X$ with respect to $\circ$ and $*$.

(v) The binary operations $\diamond$ and $\star$ on $E$ are continuous. The square operator $s \mapsto s \diamond s$ is an automorphism of $E$ with respect to $\diamond$ and $\star$. We denote by $\tilde{\tau}$ the inverse mapping of this automorphism.

(vi) $\tilde{\alpha} \equiv \alpha_{\tilde{\sigma}, \varepsilon}<\infty, \tilde{\beta} \equiv \beta_{\tilde{\sigma}, \delta}<\infty, \tilde{\gamma} \equiv \gamma_{\tilde{\tau}}<\infty$, and $\tilde{\gamma} \max \{\tilde{\alpha}, \tilde{\beta}\}<1$.

Under the above conditions, we show the Ulam type stability for the commutative diagram (1), as follows.

Theorem 2 Let $(X, \circ, *)$ and $(E, d, \diamond, \star)$ be as above. Suppose that four mappings $\tilde{\sigma}: X \rightarrow X$, $\tilde{\tau}: E \rightarrow E, \varepsilon: X \times X \rightarrow \mathbb{R}^{+}$and $\delta: X \rightarrow \mathbb{R}^{+}$satisfy (iv), (v), and (vi). If a mapping $f: X \rightarrow E$ satisfies (3) and

$$
d(f(x \circ x) \star f(x * x), f(x \circ x)) \leq \delta(x) \quad(\forall x \in X),
$$

then there exists a mapping $f_{\infty}: X \rightarrow E$ satisfying (5)

$$
\begin{aligned}
& f_{\infty}(x \circ x) \star f_{\infty}(x * x)=f_{\infty}(x \circ x) \quad(\forall x \in X), \\
& d\left(f(x), f_{\infty}(x)\right) \leq \frac{\tilde{\gamma}\{\varepsilon(x, x)+\delta(x)\}}{1-\tilde{\gamma} \max \{\tilde{\alpha}, \tilde{\beta}\}} \quad(\forall x \in X) .
\end{aligned}
$$

Moreover, if a mapping $g: X \rightarrow$ E satisfies (13), (14), and

$$
\exists K_{g} \geq 0: d(f(x), g(x)) \leq K_{g} \tilde{\gamma}\{\varepsilon(x, x)+\delta(x)\} \quad(\forall x \in X),
$$

then $g=f_{\infty}$. 
Proof For simplicity, we write $\tilde{T}=T_{\tilde{\sigma}, \tilde{\tau}}$, that is, $(\tilde{T} f)(x)=\tilde{\tau}(f(\tilde{\sigma} x))$ for $x \in X$. We note that $\tilde{\alpha}, \tilde{\beta}$ and $\tilde{\gamma}$ are finite by (vi). Suppose that $f: X \rightarrow E$ satisfies (3) and (12). Put $\tilde{\varphi}(x)=\tilde{\gamma}\{\varepsilon(x, x)+\delta(x)\}$ for all $x \in X$. To apply Lemma A to $f$ and $\tilde{\varphi}$, we first observe that $\tilde{T} f \in \Delta_{f, \tilde{\varphi}}$. Fix $x \in X$. Since $\tilde{\tau}(f(x) \diamond f(x))=f(x)$, it follows from (3) and (12) that

$$
\begin{aligned}
& d((\tilde{T} f)(x), f(x)) \\
& \quad=d(\tilde{\tau}(f(\tilde{\sigma} x)), f(x)) \\
& \quad=d(\tilde{\tau}(f(x \circ x)), \tilde{\tau}(f(x) \diamond f(x))) \\
& \quad \leq \tilde{\gamma} d(f(x \circ x), f(x) \diamond f(x)) \\
& \quad \leq \tilde{\gamma}\{d(f(x \circ x), f(x \circ x) \star f(x * x))+d(f(x \circ x) \star f(x * x), f(x) \diamond f(x))\} \\
& \quad \leq \tilde{\gamma}\{\delta(x)+\varepsilon(x, x)\} \\
& \quad=\tilde{\varphi}(x) .
\end{aligned}
$$

Hence $\tilde{T} f \in \Delta_{f, \tilde{\varphi}}$ and $\rho_{f, \tilde{\varphi}}(\tilde{T} f, f) \leq 1$.

We next estimate the quantity $\beta_{\tilde{\sigma}, \tilde{\varphi}}$. For $x \in X$, we have

$$
\begin{aligned}
\tilde{\varphi}(\tilde{\sigma} x) & =\tilde{\gamma}\{\varepsilon(\tilde{\sigma} x, \tilde{\sigma} x)+\delta(\tilde{\sigma} x)\} \\
& \leq \tilde{\gamma}\{\tilde{\alpha} \varepsilon(x, x)+\tilde{\beta} \delta(x)\} \\
& \leq \tilde{\gamma} \max \{\tilde{\alpha}, \tilde{\beta}\}\{\varepsilon(x, x)+\delta(x)\} \\
& =\max \{\tilde{\alpha}, \tilde{\beta}\} \tilde{\varphi}(x) .
\end{aligned}
$$

Hence $\beta_{\tilde{\sigma}, \tilde{\varphi}} \leq \max \{\tilde{\alpha}, \tilde{\beta}\}$ and $\beta_{\tilde{\sigma}, \tilde{\varphi}} \gamma_{\tilde{\tau}} \leq \tilde{\gamma} \max \{\tilde{\alpha}, \tilde{\beta}\}<1$ by (vi).

Thus we can apply Lemma A. As a consequence, $\tilde{T}$ has a unique fixed point $f_{\infty} \in \Delta_{f, \tilde{\varphi}}$. Moreover,

$$
\lim _{n \rightarrow \infty} d\left(\left(\tilde{T}^{n} f\right)(x), f_{\infty}(x)\right)=0
$$

and

$$
d\left(f(x), f_{\infty}(x)\right) \leq \frac{\rho_{f, \tilde{\varphi}}(\tilde{T} f, f)}{1-\beta_{\tilde{\sigma}, \tilde{\varphi} \gamma \tilde{\tau}}} \tilde{\varphi}(x)
$$

for all $x \in X$. Since $\rho_{f, \tilde{\varphi}}(\tilde{T} f, f) \leq 1$ and $\beta_{\tilde{\sigma}, \tilde{\varphi}} \gamma_{\tilde{\tau}} \leq \tilde{\gamma} \max \{\tilde{\alpha}, \tilde{\beta}\}<1$, (17) implies (14).

Here we show (5). If $x, y \in X$ and $n \in \mathbb{N}$, then we have

$$
\begin{aligned}
& d\left(f_{\infty}(x \circ y) \star f_{\infty}(x * y), f_{\infty}(x) \diamond f_{\infty}(y)\right) \\
& \leq d\left(f_{\infty}(x \circ y) \star f_{\infty}(x * y),\left(\tilde{T}^{n} f\right)(x \circ y) \star\left(\tilde{T}^{n} f\right)(x * y)\right) \\
&+d\left(\left(\tilde{T}^{n} f\right)(x \circ y) \star\left(\tilde{T}^{n} f\right)(x * y),\left(\tilde{T}^{n} f\right)(x) \diamond\left(\tilde{T}^{n} f\right)(y)\right) \\
&+d\left(\left(\tilde{T}^{n} f\right)(x) \diamond\left(\tilde{T}^{n} f\right)(y), f_{\infty}(x) \diamond f_{\infty}(y)\right) .
\end{aligned}
$$

Letting $n \rightarrow \infty$, the first and third terms on the right hand side tend to 0 , because of (16) and the continuity of $\star$ and $\diamond$ in (v). Moreover, the second term, say $\tilde{A}_{n}(x, y)$, is estimated 
as follows: By (iv), (v), and (3),

$$
\begin{aligned}
\tilde{A}_{n}(x, y) & =d\left(\tilde{\tau}^{n}\left(f\left(\tilde{\sigma}^{n}(x \circ y)\right)\right) \star \tilde{\tau}^{n}\left(f\left(\tilde{\sigma}^{n}(x * y)\right)\right), \tilde{\tau}^{n}\left(f\left(\tilde{\sigma}^{n} x\right)\right) \diamond \tilde{\tau}^{n}\left(f\left(\tilde{\sigma}^{n} y\right)\right)\right) \\
& =d\left(\tilde{\tau}^{n}\left(f\left(\tilde{\sigma}^{n} x \circ \tilde{\sigma}^{n} y\right)\right) \star \tilde{\tau}^{n}\left(f\left(\tilde{\sigma}^{n} x * \tilde{\sigma}^{n} y\right)\right), \tilde{\tau}^{n}\left(f\left(\tilde{\sigma}^{n} x\right) \diamond f\left(\tilde{\sigma}^{n} y\right)\right)\right) \\
& =d\left(\tilde{\tau}^{n}\left(f\left(\tilde{\sigma}^{n} x \circ \tilde{\sigma}^{n} y\right) \star f\left(\tilde{\sigma}^{n} x * \tilde{\sigma}^{n} y\right)\right), \tilde{\tau}^{n}\left(f\left(\tilde{\sigma}^{n} x\right) \diamond f\left(\tilde{\sigma}^{n} y\right)\right)\right) \\
& \leq \tilde{\gamma}^{n} d\left(f\left(\tilde{\sigma}^{n} x \circ \tilde{\sigma}^{n} y\right) \star f\left(\tilde{\sigma}^{n} x * \tilde{\sigma}^{n} y\right), f\left(\tilde{\sigma}^{n} x\right) \diamond f\left(\tilde{\sigma}^{n} y\right)\right) \\
& \leq \tilde{\gamma}^{n} \varepsilon\left(\tilde{\sigma}^{n} x, \tilde{\sigma}^{n} y\right) \\
& \leq \tilde{\gamma}^{n} \tilde{\alpha}^{n} \varepsilon(x, y),
\end{aligned}
$$

where $\tilde{\tau}^{n}$ and $\tilde{\sigma}^{n}$ denote the $n$-fold compositions of endomorphisms $\tilde{\tau}$ and $\tilde{\sigma}$, respectively. Since $\tilde{\gamma} \tilde{\alpha}<1$ by (vi), it follows that $\tilde{A}_{n}(x, y) \rightarrow 0$ as $n \rightarrow \infty$. Thus we obtain (5).

Next, we show (13). Replacing $y$ in (5) by $x$, we have

$$
f_{\infty}(x \circ x) \star f_{\infty}(x * x)=f_{\infty}(x) \diamond f_{\infty}(x)
$$

Also since

$$
\tilde{\tau}\left(f_{\infty}(x \circ x)\right)=\tilde{\tau}\left(f_{\infty}(\tilde{\sigma} x)\right)=\left(\tilde{T} f_{\infty}\right)(x)=f_{\infty}(x)=\tilde{\tau}\left(f_{\infty}(x) \diamond f_{\infty}(x)\right)
$$

it follows that

$$
f_{\infty}(x \circ x)=f_{\infty}(x) \diamond f_{\infty}(x)
$$

Combining with (18), we obtain (13).

Finally, we show the last statement. Since $g$ satisfies (14) and (13), we have

$$
g(\tilde{\sigma} x)=g(x \circ x)=g(x \circ x) \star g(x * x)=g(x) \diamond g(x)=\tilde{\tau}^{-1}(g(x)),
$$

that is, $(\tilde{T} g)(x)=g(x)$ for all $x \in X$. This says that $g$ is a fixed point of $\tilde{T}$. Also, by (15), we have $g \in \Delta_{f, \tilde{\varphi}}$. Hence the uniqueness of a fixed point of $\tilde{T}$ in $\Delta_{f, \tilde{\varphi}}$ implies that $g=f_{\infty}$.

The next corollary is obtained in [6].

Corollary 2 ([6, Corollary 3.5]) Let $X$ be a set with a binary operation $\circ$ such that the square operation $\tilde{\sigma}: x \mapsto x \circ x$ is an endomorphism of $X$ with respect to $\circ$ and $E$ a complete metric space with a continuous binary operation $\diamond$ such that the square operation $s \mapsto$ $s \diamond s$ is an automorphism of $E$ with respect to $\diamond$. Let $\varepsilon: X \times X \rightarrow \mathbb{R}^{+}$and suppose that $\tilde{\alpha} \equiv \alpha_{\tilde{\sigma}, \varepsilon}<\infty, \tilde{\gamma} \equiv \gamma_{\tilde{\tau}}<\infty$ and $\tilde{\gamma} \tilde{\alpha}<1$, where $\tilde{\tau}$ denotes the inverse mapping of the square operation $s \mapsto s \diamond$ s. If a mapping $f: X \rightarrow E$ satisfies

$$
d(f(x \circ y), f(x) \diamond f(y)) \leq \varepsilon(x, y) \quad(\forall x, y \in X),
$$

then there exists a unique mapping $f_{\infty}: X \rightarrow E$ such that

$$
f_{\infty}(x \circ y)=f_{\infty}(x) \diamond f_{\infty}(y) \quad \text { and } \quad d\left(f(x), f_{\infty}(x)\right) \leq \frac{\tilde{\gamma}}{1-\tilde{\alpha} \tilde{\gamma}} \varepsilon(x, x)
$$

for all $x, y \in X$. 
Proof Consider the case that $*=\circ$ and $s \star t=s$ for $s, t \in E$, in Theorem 2. Then $\tilde{\tau}$ is clearly an endomorphism of $E$ with respect to $\star$. Therefore the corollary follows immediately from Theorem 2 with $\delta=0$.

\section{Application I}

The Ulam type stability problem for Euler-Lagrange type additive mappings has been investigated in [17]. Here we take up the following Euler-Lagrange type mapping $f: X \rightarrow E$ satisfying

$$
f(a x+b y)+f(b x+a y)+(a+b)(f(-x)+f(-y))=0 \quad(\forall x, y \in X),
$$

where $X$ is a complex normed space, $E$ a complex Banach space and $a, b \in \mathbb{C}$ with $a+b \neq 0$. The following is an Ulam type stability result for this mapping.

Corollary 3 (cf. [17, Theorem 2.1]) Let $\varepsilon: X \times X \rightarrow \mathbb{R}^{+}$and suppose that

(vii) $\exists K \geq 0:|a+b| K<1$ and $\varepsilon(x, y) \leq K \varepsilon(-(a+b) x,-(a+b) y)(\forall x, y \in X)$.

If a mapping $f: X \rightarrow$ E satisfies

$$
\|f(a x+b y)+f(b x+a y)+(a+b)(f(-x)+f(-y))\| \leq \varepsilon(x, y) \quad(\forall x, y \in X),
$$

then there exists a unique mapping $f_{\infty}: X \rightarrow E$ satisfying (19) and

$$
\left\|f(x)-f_{\infty}(x)\right\| \leq \frac{K}{2(1-|a+b| K)} \varepsilon(-x,-x) \quad(\forall x \in X) .
$$

Proof Put $u=-x, v=-y$ for each $x, y \in X$. Under these transformations, (20) changes into the following estimate:

$$
\left\|\frac{1}{2}\{f(-a u-b v)+f(-b u-a v)\}+\frac{a+b}{2}\{f(u)+f(v)\}\right\| \leq \varepsilon_{1}(u, v) \quad(\forall u, v \in X),
$$

where $\varepsilon_{1}(u, v)=\frac{1}{2} \varepsilon(-u,-v)(\forall u, v \in X)$.

Now we define $u \circ v=-a u-b v, u * v=-b u-a v$ for each $u, v \in X$. In this case, we can easily see that the square operator $u \mapsto u \circ u$ is an endomorphism of $X$ with respect to $\circ$ and $*$. Also since $a+b \neq 0$, this endomorphism is bijective and so automorphic. We denote by $\sigma$ the inverse mapping of this automorphism. Moreover, we define $s \diamond t=-\frac{1}{2}(a+b)(s+t)$, $s \star t=\frac{1}{2}(s+t)$ for each $s, t \in E$. Then we can also see that the binary operations $\diamond$ and $\star$ on $E$ are continuous and the square operator $\tau: s \mapsto s \diamond s$ is an automorphism of $E$ with respect to $\diamond$ and $\star$. Note that (22) changes into the following:

$$
\|f(u \circ v) \star f(u * v)-f(u) \diamond f(v)\| \leq \varepsilon_{1}(u, v) \quad(\forall u, v \in X) .
$$

Since $x \circ x=x * x$ for all $x \in X$, it follows that $\sigma x * \sigma x=\sigma x \circ \sigma x=\sigma^{-1} \sigma x=x$ for all $x \in X$. Also, since $s \star s=s$ for all $s \in E$, it follows that $f(x) \star f(\sigma x * \sigma x)=f(x) \star f(x)=f(x)$ for all $x \in X$ and then (4) holds with $\delta=0$. Moreover, $\beta_{\sigma, \delta}=0$ must hold with $\delta=0$. It is also obvious that $\gamma_{\tau}=|a+b|$ from the definition of $\tau$. We also note that $\alpha_{\sigma, \varepsilon_{1}} \leq K$ from the 
second condition of (vii) and hence $\gamma_{\tau} \alpha_{\sigma, \varepsilon_{1}} \leq|a+b| K<1$ from the first condition of (vii). Therefore, by Theorem 1, there exists a unique mapping $f_{\infty}: X \rightarrow E$ such that

$$
f_{\infty}(u \circ v) \star f_{\infty}(u * v)=f_{\infty}(u) \diamond f_{\infty}(v) \quad(\forall u, v \in X),
$$

namely, (19) holds and

$$
\left\|f(u)-f_{\infty}(u)\right\| \leq \frac{\alpha_{\sigma, \varepsilon_{1}} \varepsilon_{1}(u, u)}{1-\gamma_{\tau} \max \left\{\alpha_{\sigma, \varepsilon_{1}}, \beta_{\sigma, \delta}\right\}} \leq \frac{K}{2(1-|a+b| K)} \varepsilon(-u,-u) \quad(\forall u \in X),
$$

and so (21) holds.

The following is also an Ulam type stability result for the mapping satisfying (19).

Corollary 4 (cf. [17, Theorem 2.2]) Let $\varepsilon: X \times X \rightarrow \mathbb{R}^{+}$and suppose that

(viii) $\exists K \geq 0: K<|a+b|$ and $\varepsilon(-(a+b) x,-(a+b) y) \leq K \varepsilon(x, y)(\forall x, y \in X)$.

If a mapping $f: X \rightarrow E$ satisfies (20), then there exists a unique mapping $f_{\infty}: X \rightarrow E$ satisfying (19) and

$$
\left\|f(x)-f_{\infty}(x)\right\| \leq \frac{1}{2(|a+b|-K)} \varepsilon(-x,-x) \quad(\forall x \in X) .
$$

Proof As observed in the proof of Corollary 3, (20) changes into (22). Now we define $u \circ v=-a u-b v, u * v=-b u-a v$ for each $u, v \in X$. In this case, we can easily see that the square operator $\tilde{\sigma}: u \mapsto u \circ u$ is an endomorphism of $X$ with respect to $\circ$ and $*$. Moreover, we define $s \diamond t=-\frac{1}{2}(a+b)(s+t), s \star t=\frac{1}{2}(s+t)$ for each $s, t \in E$. Then we can also see that the binary operations $\diamond$ and $\star$ on $E$ are continuous and the square operator $s \mapsto s \diamond s$ is an endomorphism of $E$ with respect to $\diamond$ and $\star$. Also since $a+b \neq 0$, this endomorphism is bijective and so automorphic. We denote by $\tilde{\tau}$ the inverse mapping of this automorphism. Note that (22) changes into (23). Since $x \circ x=x * x(\forall x \in X)$ and $s \star s=s(\forall s \in E)$, it follows that $f(x \circ x) \star f(x * x)=f(x \circ x)$ for all $x \in X$ and then (12) holds with $\delta=0$.

Moreover, $\beta_{\tilde{\sigma}, \delta}=0$ must hold with $\delta=0$. It is also obvious that $\gamma_{\tilde{\tau}}=|a+b|^{-1}$ from the definition of $\tilde{\tau}$. We also note that $\alpha_{\tilde{\sigma}, \varepsilon_{1}} \leq K$ from the second condition of (viii) and hence $\gamma_{\tilde{\tau}} \alpha_{\tilde{\sigma}, \varepsilon_{1}} \leq|a+b|^{-1} K<1$ from the first condition of (viii).

Therefore, by Theorem 2 , there exists a unique mapping $f_{\infty}: X \rightarrow E$ such that

$$
f_{\infty}(u \circ v) \star f_{\infty}(u * v)=f_{\infty}(u) \diamond f_{\infty}(v) \quad(\forall u, v \in X),
$$

namely, (19) holds and

$$
\begin{aligned}
\left\|f(u)-f_{\infty}(u)\right\| & \leq \frac{\gamma_{\tilde{\tau}} \varepsilon_{1}(u, u)}{1-\gamma_{\tilde{\tau}} \max \left\{\alpha_{\tilde{\sigma}, \varepsilon_{1}}, \beta_{\tilde{\sigma}, \delta}\right\}} \\
& \leq \frac{|a+b|^{-1}}{2\left(1-|a+b|^{-1} K\right)} \varepsilon(-u,-u) \\
& =\frac{1}{2(|a+b|-K)} \varepsilon(-u,-u) \quad(\forall u \in X),
\end{aligned}
$$

and so (24) holds. 
Corollary 5 (cf. [17, Corollary 2.3]) Suppose that $|a+b| \neq 1, \delta, p, q \geq 0$ and $p+q \neq 1$. If $a$ mapping $f: X \rightarrow$ E satisfies

$$
\|f(a x+b y)+f(b x+a y)+(a+b)\{f(-x)+f(-y)\}\| \leq \delta\|x\|^{p}\|y\|^{q}
$$

for all $x, y \in X$, then there exists a unique mapping $f_{\infty}: X \rightarrow E$ satisfying (19) and

$$
\left\|f(x)-f_{\infty}(x)\right\| \leq \frac{\delta}{2\left(\left\|a+\left.b\right|^{p+q}-|a+b \||\right.\right.}\|x\|^{p+q} \quad(\forall x \in X) .
$$

Proof Put $\varepsilon(x, y)=\delta\|x\|^{p}\|y\|^{q}$ for each $x, y \in X$.

(a) The case where either

$$
\left\{\begin{array}{c}
|a+b|>1, \\
p+q>1
\end{array}\right.
$$

or

$$
\left\{\begin{array}{c}
|a+b|<1 \\
p+q<1
\end{array}\right.
$$

Put $K=|a+b|^{-(p+q)}$. Then $K$ satisfies (vii). Note also that

$$
\frac{K}{2(1-|a+b| K)} \varepsilon(-x,-x)=\frac{\delta}{2\left(|a+b|^{p+q}-|a+b|\right)}\|x\|^{p+q}
$$

for all $x \in X$. Then the desired result follows from Corollary 3 .

(b) The case where either

$$
\left\{\begin{array}{c}
|a+b|>1, \\
p+q<1,
\end{array}\right.
$$

or

$$
\left\{\begin{array}{c}
|a+b|<1 \\
p+q>1
\end{array}\right.
$$

Put $K=|a+b|^{p+q}$. Then $K$ satisfies (viii). Note also that

$$
\frac{1}{2(|a+b|-K)} \varepsilon(-x,-x)=\frac{\delta}{2\left(|a+b|-|a+b|^{p+q}\right)}\|x\|^{p+q}
$$

for all $x \in X$. Then the desired result follows from Corollary 4 .

\section{Application II}

Let $(X,+)$ be an Abelian group. In [18], the following result has been shown by A. Simon and P. Volkmann. 
Lemma B ([18, Théorème 1)] A mapping $f: X \rightarrow \mathbb{R}$ satisfies

$$
\max \{f(x+y), f(x-y)\}=f(x)+f(y) \quad(\forall x, y \in X),
$$

if and only if $f(x)=|\pi(x)|(\forall x \in X)$ for some additive function $\pi: X \rightarrow \mathbb{R}$.

In this section, we deal with the Ulam type stability problem for Equation (25). Put $x \circ y=$ $x+y$ and $x * y=x-y$ for each $x, y \in X$. Moreover, put $s \diamond t=s+t$ and $s \star t=\max \{s, t\}$ for each $s, t \in \mathbb{R}$. Then (25) changes into (2). Also we can easily see that the square operation $\tilde{\sigma}: x \mapsto x \circ x$ is endomorphic with respect to $\circ$ and $*$ and that the square operator $s \mapsto$ $s \diamond s$ is automorphic with respect to $\diamond$ and $\star$. Denote by $\tilde{\tau}$ the inverse mapping of this automorphism. In this case, it is obvious that $\tilde{\tau}(s)=\frac{1}{2} s$ for each $s \in \mathbb{R}$ and hence $\gamma_{\tilde{\tau}}=1 / 2$.

Now let $\varepsilon$ be a nonnegative constant and suppose that $f: X \rightarrow \mathbb{R}$ satisfies

$$
|\max \{f(x+y), f(x-y)\}-\{f(x)+f(y)\}| \leq \varepsilon \quad(\forall x, y \in X) .
$$

Putting $x=y=0$ in (26), we obtain

$$
|f(0)| \leq \varepsilon
$$

Also, putting $x=y$ in (26), we obtain

$$
-\varepsilon+f(0) \leq-\varepsilon+\max \{f(x+x), f(0)\} \leq 2 f(x) \quad(\forall x \in X) .
$$

Combining (27) and (28), we obtain

$$
-\varepsilon \leq f(x) \quad(\forall x \in X)
$$

Put $\delta=2 \varepsilon$. By (27) and (28), we obtain

$$
0 \leq \max \{f(x+x), f(0)\}-f(x+x) \leq \varepsilon+\varepsilon=\delta \quad(\forall x \in X),
$$

and hence (12) holds. Moreover, note that $\alpha_{\tilde{\sigma}, \varepsilon}=\beta_{\tilde{\sigma}, \delta}=1$ since $\varepsilon$ and $\delta$ are constant. Then Lemma B and Theorem 2 easily imply the following.

Corollary 6 Let $X$ be an Abelian group and $\varepsilon$ a nonnegative constant. If $: X \rightarrow \mathbb{R}$ satisfies (26), then there exists an additive mapping $\pi: X \rightarrow \mathbb{R}$ such that

$$
|f(x)-| \pi(x)|| \leq 3 \varepsilon \quad(\forall x \in X) .
$$

For the related results, see $[19,20]$. 


\section{Author details}

${ }^{1}$ Department of Information Sciences, Toho University, Funabashi, 274-8501, Japan. ${ }^{2}$ Department of Mathematics, Niigata University, Niigata, 950-2181, Japan. ${ }^{3}$ Department of Mathematical Sciences, Faculty of Science, Shinshu University,

Matsumoto, 390-8621, Japan. ${ }^{4}$ Department of Mathematics, Tohoku Pharmaceutical University, Sendai, 981-8558, Japan.

\section{Acknowledgements}

The authors are deeply grateful to the referees for careful reading of the paper and for the helpful suggestions and comments. All authors are partially supported by Grant-in-Aid for Scientific Research, Japan Society for the Promotion of Science.

\section{Received: 29 January 2014 Accepted: 27 May 2014 Published: 04 Jun 2014}

\section{References}

1. Brillouët-Belluot, N, Brzdęk, J, Ciepliński, K: On some recent developments in Ulam's type stability. Abstr. Appl. Anal. 2012, Article ID 716936 (2012)

2. Brzdęk, J: Hyperstability of the Cauchy equation on restricted domains. Acta Math. Hung. 141, 58-67 (2013)

3. Brzdęk, J, Ciepliński, K: Hyperstability and superstability. Abstr. Appl. Anal. 2013, Article ID 401756 (2013)

4. Gajda, Z: On stability of additive mappings. Int. J. Math. Math. Sci. 14, 431-434 (1991)

5. Jung, S-M: Hyers-Ulam-Rassias Stability of Functional Equations in Nonlinear Analysis. Springer Optimization and Its Applications, vol. 48. Springer, New York (2011)

6. Takahasi, S-E, Miura, T, Takagi, H: On a Hyers-Ulam-Aoki-Rassias type stability and a fixed point theorem. J. Nonlinear Convex Anal. 11, 423-439 (2010)

7. Bahyrycz, A, Piszczek, M: Hyperstability of the Jensen functional equation. Acta Math. Hung. 142, 353-365 (2014)

8. Brzdęk, J: Stability of the equation of the $p$-Wright affine functions. Aequ. Math. 85, 497-503 (2013)

9. Brzdęk, J: A hyperstability result for the Cauchy equation. Bull. Aust. Math. Soc. 89, 33-40 (2014)

10. Gilányi, A, Kaiser, Z, Páles, Z: Estimates to the stability of functional equations. Aequ. Math. 73, 125-143 (2007)

11. Kim, GH: On the stability of functional equations with square-symmetric operation. Math. Inequal. Appl. 4, 257-266 (2001)

12. Kim, GH: Addendum to 'On the stability of functional equations on square-symmetric groupoid'. Nonlinear Anal. 62 , 365-381 (2005)

13. Páles, Z: Hyers-Ulam stability of the Cauchy functional equation on square-symmetric groupoids. Publ. Math. (Debr.) 58, 651-666 (2001)

14. Páles, Z, Volkmann, P, Luce, RD: Hyers-Ulam stability of functional equations with square symmetric operations. Proc. Natl. Acad. Sci. USA 95, 12772-12775 (1998)

15. Piszczek, M: Remark on hyperstability of the general linear equation. Aequ. Math. (2013). doi:10.1007/s00010-013-0214-x

16. Ciepliński, K: Applications of fixed point theorems to the Hyers-Ulam stability of functional equations-a survey. Ann. Funct. Anal. 3, 151-164 (2012)

17. Kim, H-M, Jun, K-W, Rassias, JM: Extended stability problem for alternative Cauchy-Jensen mappings. J. Inequal. Pure Appl. Math. 8, 120 (2007)

18. Simon, A, Volkmann, P: Caractérisation du module d'une fonction á l'aide d'une équation fonctionnelle. Aequ. Math 47, 60-68 (1994)

19. Gilányi, A, Nagatou, K, Volkmann, P: Stability of a functional equation coming from the characterization of the absolute value of additive functions. Ann. Funct. Anal. 1, 1-6 (2010)

20. Jarczyk, W, Volkmann, P: On functional equations in connection with the absolute value of additive functions. Series Mathematicae Catoviciensis et Debreceniensis 32, 1-11 (2010)

10.1186/1029-242X-2014-228

Cite this article as: Takahasi et al.: Ulam type stability problems for alternative homomorphisms. Journal of Inequalities and Applications 2014, 2014:228

\section{Submit your manuscript to a SpringerOpen ${ }^{\ominus}$ journal and benefit from:}

- Convenient online submission

- Rigorous peer review

Immediate publication on acceptance

Open access: articles freely available online

- High visibility within the field

- Retaining the copyright to your article 